\title{
Acceleration of callus formation during fracture healing using basic fibroblast growth factor-kidney disease domain-collagen-binding domain fusion protein combined with allogenic demineralized bone powder
}

Wataru Saito ${ }^{1}$, Kentaro Uchida ${ }^{1 *}$, Osamu Matsushita², Gen Inoue ${ }^{1}$, Hiroyuki Sekiguchi ${ }^{1}$, Jun Aikawa', Hisako Fujimaki ${ }^{1}$ and Masashi Takaso ${ }^{1}$

\begin{abstract}
Background: To repair fractures with large bone defects or gaps, demineralized allogenic bone matrix (DBM) is often applied to the fracture site. However, studies have shown that the use of DBM alone has limited efficacy for repairing fractures. In the present study, we developed an allogenic demineralized bone powder (DBP) with basic fibroblast-derived growth factor containing a polycystic kidney disease (PKD) domain and collagen-binding domain (CBD) from Clostridium histolyticum collagenase $(\mathrm{ColH})$ and investigated the stimulatory effects of bFGF-PKD-CBD combined with allogenic DBP on bone growth in a mouse femur fracture model.

Methods: DBP mixed with either phosphate-buffered saline (PBS) (DBP/PBS), 0.58 nmol basic fibroblast growth factor (bFGF) (0.58 nmol DBP/bFGF), $0.058 \mathrm{nmol}$ bFGF-PKD-CBD (0.058 nmol DBP/bFGF-PKD-CBD), or $0.58 \mathrm{nmol}$ bFGF-PKD-CBD (0.58 nmol DBP/bFGF-PKD-CBD) was grafted into fracture sites.

Results: bFGF-PKD-CBD/DBP composite accelerates callus formation in a bone fracture model in mice and clearly showed that the composite also increases bone mineral density at fracture sites compared to bFGF/DBP. In addition, bFGF-PKD-CBD/DBP increased callus volume and bone mineral content to similar levels in fractures treated with a tenfold higher amount of bFGF at 4 weeks.
\end{abstract}

Conclusions: Our results suggest that bFGF-PKD-CBD/DBP may be useful for promoting fracture healing in the clinical setting.

Keywords: Bone powder, Basic fibroblast growth factor, Collagen-binding domain, Fracture healing,

Allogenic demineralized bone matrix

\section{Background}

Certain types of fractures are associated with delayed union or nonunion and therefore require bone grafting [1-3]. Allogenic demineralized bone matrix (DBM) is a useful bone-filling material because it serves as biologic osteoconductive scaffold that retains the trabecular collagenous structure of the original tissue, and has bone

\footnotetext{
* Correspondence: kuchida@med.kitasato-u.ac.jp

1 Department of Orthopaedic Surgery, Kitasato University School of Medicine,

1-15-1 Minami-ku Kitasato, Sagamihara, Kanagawa, Japan

Full list of author information is available at the end of the article
}

morphogenetic protein 2 (BMP-2) retention/liberation properties $[4,5]$. Due to these properties, DBM is one of the most extensively used bone scaffolds in the clinical setting worldwide [1,6]. However, studies have shown that the use of DBM alone has limited efficacy for repairing bone defects $[7,8]$.

To overcome this limitation, strategies for the repair of large fractures with DBM should include boneinducing growth factors. A number of growth factors, such as BMP-2, basic fibroblast growth factor (bFGF), and osteogenic protein-1 (OP-1), have been studied for

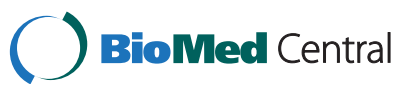

(c) 2015 Saito et al.; licensee BioMed Central. This is an Open Access article distributed under the terms of the Creative Commons Attribution License (http://creativecommons.org/licenses/by/4.0), which permits unrestricted use, distribution, and reproduction in any medium, provided the original work is properly credited. The Creative Commons Public Domain Dedication waiver (http://creativecommons.org/publicdomain/zero/1.0/) applies to the data made available in this article, unless otherwise stated. 
their potential to promote bone growth and union [9-14]. For example, BMP-2 and bFGF have been shown to accelerate the healing of fractures and bone defects $[11,12]$, and OP-1 has been successfully used to treat tibial nonunions $[9,10]$. However, in practice, bone-inducing factors rapidly diffuse in body fluid and fall below therapeutic concentrations at defect sites. For this reason, large doses and/or repeated administrations of growth factors are required for sustained therapeutic effect, but such dosing regimens may be clinically impractical and expensive and can lead to adverse side effects $[15,16]$. To increase the osteogenic potential of bone-inducing growth factors, they should ideally be combined with a carrier, such as collagen or DBM, to promote their retention at fracture sites.

We previously fused the collagen-binding domain (CBD) and polycystic kidney disease (PKD) domain of Clostridium histolyticum class II collagenase $(\mathrm{ColH})$ to bFGF and showed that the subcutaneous injection of this collagen-binding bFGF fusion protein (bFGF-PKD-CBD) without carrier into nude mice had more potent skin fibroblast growth-promoting effects at the injection site than native bFGF [17]. bFGF-PKD-CBD also markedly enhanced bone formation when loaded onto autologous DBM that was grafted onto intact rat femurs [18]. Based on these findings, we speculated that the combination of bFGF-PKD-CBD and DBM may promote the retention of bFGF at injury sites and thereby accelerate bone repair. However, the efficacy of this treatment approach has only been evaluated with autologous DBM and healthy bone, and the bone formation-promoting effects of bFGF-PKD-CBD in combination with allogenic DBM in bone injury models have not been determined.

Here, we investigated the stimulatory effects of bFGFPKD-CBD combined with allogenic demineralized bone powder (DBP) on bone growth in a mouse femur fracture model.

\section{Methods}

Preparation of allogenic dematerialized bone powder

Both femurs were harvested from $36 \mathrm{C} 3 \mathrm{H} / \mathrm{HeN}(\mathrm{H}-2 \mathrm{k})$ mice, and bone lipids were removed by treatment with chloroform/methanol. The harvested femoral bones were broken into small fragments, which were then passed through a $1-\mathrm{mm}$ filter to collect the bone powder. To prepare DBP, the bone powder was demineralized using 0.6 $\mathrm{N} \mathrm{HCl}$ for $18 \mathrm{~h}$ at $4^{\circ} \mathrm{C}$. The particle size distributions were determined by laser scattering using a LMS-30 Micron Sizer (Seishin Enterprise Co., Ltd., Tokyo, Japan) and cumulative size distribution limits of D10, D50, and D90, which correspond to the percentage of particles $(10 \%$, $50 \%$, and $90 \%$, respectively) in a sample that is below a certain size. The surfaces of the DBP were observed by scanning transmission electron microscopy (SEM; JSM7400F; JEOL Ltd., Tokyo, Japan).

\section{Preparation of bFGF and bFGF-PKD-CBD}

Recombinant human bFGF was purchased from Kaken Pharmaceuticals (Tokyo, Japan). The construction of the fusion protein of bFGF, PKD, and the CBD derived from C. histolyticum class II collagenase $(\mathrm{ColH})$ was previously described [18]. The biological activities of purified bFGFPKD-CBD were confirmed using a proliferation assay with cultured periosteal mesenchymal cells [19]. bFGF-PKDCBD exhibited the same cell proliferation ability as bFGF in vitro. The affinity of bFGF-PKD-CBD for allogenic DBP was confirmed in vitro by ELISA, as previously described [18]. In the assay, $0.064 \mathrm{nmol}$ of bFGF-PKD-CBD bound to $1 \mathrm{mg}$ of allogenic DBP.

\section{Fracture generation}

All procedures involving the handling of animals adhered to the guidelines of the animal ethics committee of Kitasato University. A specific pathogen-free colony of $\mathrm{C} 57 \mathrm{BL} / 6 \mathrm{~J}$ mice was housed in a semi-barrier system under controlled conditions (temperature, $23^{\circ} \mathrm{C} \pm 2{ }^{\circ} \mathrm{C}$; humidity, $55 \% \pm 10 \%$; and lighting, 12 -h light/dark cycle) throughout the study at Nippon Charles River Laboratories (Kanagawa, Japan). Mice were allowed access to standard rodent chow (CRF-1; Oriental Yeast Co., Ltd., Tokyo, Japan) and water ad libitum. Fractures were generated in the femurs of $72 \mathrm{C} 57 \mathrm{BL} / 6 \mathrm{~J}$ mice by first making a 4-mm medial parapatellar incision in the left knee under sterile conditions to laterally dislocate the patella [20]. A $0.5-\mathrm{mm}$ hole was then drilled into the intracondylar notch, into which a nail $(0.5 \mathrm{~mm}$ in diameter) was inserted retrograde for the creation of fractures, which were generated with a wire saw $(0.22 \mathrm{~mm}$ in diameter $)$ using a small lateral approach. After performing the fracture generation procedure, the nail was implanted deeply to stabilize the fracture. Soft x-rays were used to confirm the successful creation of fractures [20]. As we previously demonstrated that the combination of $0.58 \mathrm{nmol}$ bFGF-PKD-CBD and collagen powder accelerated bone formation in mice [19], in the present study, the C57BL/6J mice were divided into four groups (18 mice/group), $8 \mathrm{mg}$ DBP mixed with either phosphate-buffered saline (PBS) (DBP/PBS, control group), $0.58 \mathrm{nmol}$ bFGF (0.58 nmol DBP/bFGF group), $0.058 \mathrm{nmol}$ bFGF-PKD-CBD (0.058 nmol DBP/bFGFPKD-CBD group), or $0.58 \mathrm{nmol}$ bFGF-PKD-CBD (0.58 nmol DBP/bFGF-PKD-CBD group) was grafted into fracture sites. The mice were allowed to use their fractured leg without restriction immediately after the grafting procedure until micro-CT analysis was performed at 2, 4, and 6 weeks ( $n=6$, each time point).

\section{Quantification of the mineral content and volume of newly formed callus}

Femurs and the surrounding muscle were excised from sacrificed mice at 2, 4, and 6 weeks after fracture 
generation and treatment and were then stored in $4 \%$ paraformaldehyde for $48 \mathrm{~h}$ at $4^{\circ} \mathrm{C}$. Micro-CT images of whole femurs in PBS were obtained using a microfocus X-ray CT system (inspeXio SMX-90CT; Shimadzu Co., Ltd., Tokyo, Japan) and the following settings: acceleration voltage, $90 \mathrm{kV}$; current, $110 \mathrm{~mA}$; voxel size, 20 $\mu \mathrm{m} /$ pixel; and matrix size, $1,024 \times 1,024$. From the obtained images, $10-\mathrm{mm}$ regions of interest (500 slices) in the midfemur were defined. Three-dimensional (3D) image analysis software (Tri-3D-Bon; Ratoc System Engineering Co., Ltd, Tokyo, Japan) was used to measure new bone volume and bone mineral content in the defined regions, as previously described [21]. For assessing new bone volume and bone mineral content, a hydroxyapatite (HA) calibration curve was generated from the data obtained from phantom images prepared with 200, 300, 400, 500, 600, 700, and $800 \mathrm{mg} \mathrm{HA} / \mathrm{cm}^{3}$. The bone mineral content in each sample was determined by comparing the measured densities in the micro-CT images to the HA calibration curve, and new bone was defined using a threshold value of $\geq 300 \mathrm{mg} / \mathrm{cm}^{3}$.

\section{Statistical analysis}

Differences among the PBS, bFGF, and bFGF-PKD-CBD treatment groups were examined using one-way ANOVA with the Fisher's least significant difference test. All statistical analyses were performed using SPSS software
(Version 11.0; SPSS, Inc., Chicago, IL, USA). A $P$ value of $<0.05$ was considered statistically significant.

\section{Results}

\section{Characterization of allogenic DBP}

The prepared allogenic DBP was morphologically characterized by SEM, which showed that the particles appeared irregular in shape and had a rough surface (Figure 1A, B). Based on measurements of the diameter of the bone particles, the values for D10, D50, and D90 were determined to be $154.32,266.11$, and $439.94 \mu \mathrm{m}$, respectively (Figure $1 \mathrm{C}$ ).

\section{Effect of bFGF-PKD-CBD-loaded DBP on callus formation}

Callus formation at fracture sites in mice femurs grafted with DBP in PBS (PBS/DBP) or DBP loaded with bFGF (bFGF/DBP) or bFGF-PKD-CBD (0.058 nmol and 0.58 nmol bFGF-PKD-CBD/DBP) was evaluated after 2, 4, and 6 weeks by micro-CT imaging (Figure 2A-F). Similar amounts of callus formation and bone mineral content were observed for femurs treated with PBS/DBP, bFGF/DBP, 0.058 nmol bFGF-PKD-CBD/DBP, and 0.58 nmol bFGF-PKD-CBD/DBP at 2 weeks (Figure 2E, F). However, after 4 weeks, fracture sites grafted with 0.58 nmol bFGF/DBP and 0.058 or 0.58 nmol bFGF-PKD$\mathrm{CBD} / \mathrm{DBP}$ had significantly higher levels of callus volume and bone mineral content compared to those grafted with
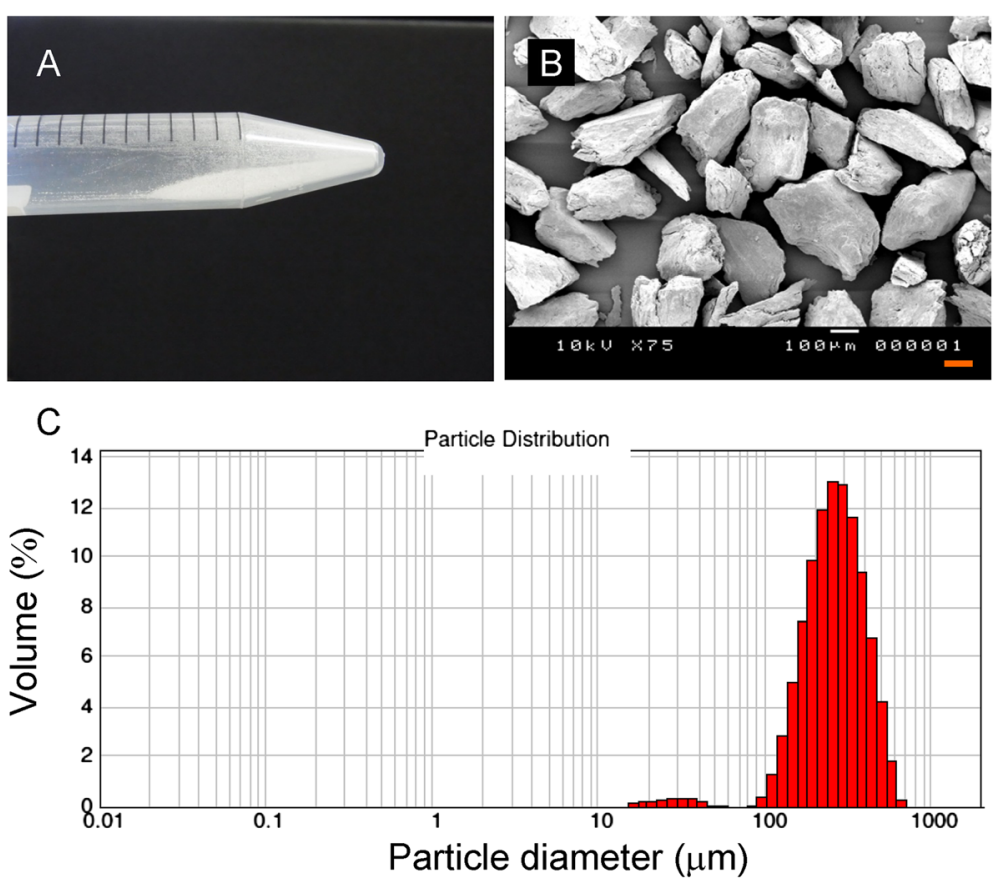

Figure 1 Appearance and SEM images of prepared demineralized allogenic bone powder. (A) Photograph of the prepared bone powder in a 1.5-cm diameter tube. (B) SEM image of the prepared bone powder. Scale bar indicates $100 \mu \mathrm{m}$. (C) Graph showing the size distribution of the prepared demineralized allogenic bone powder particles. 

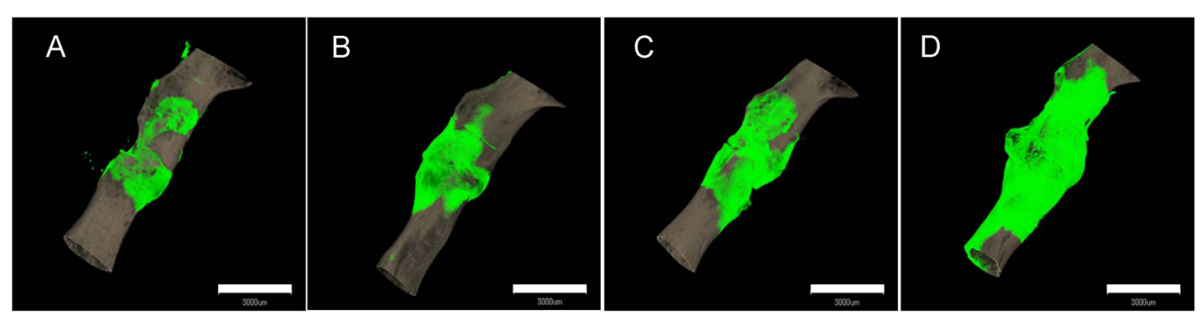

$\mathrm{E}$

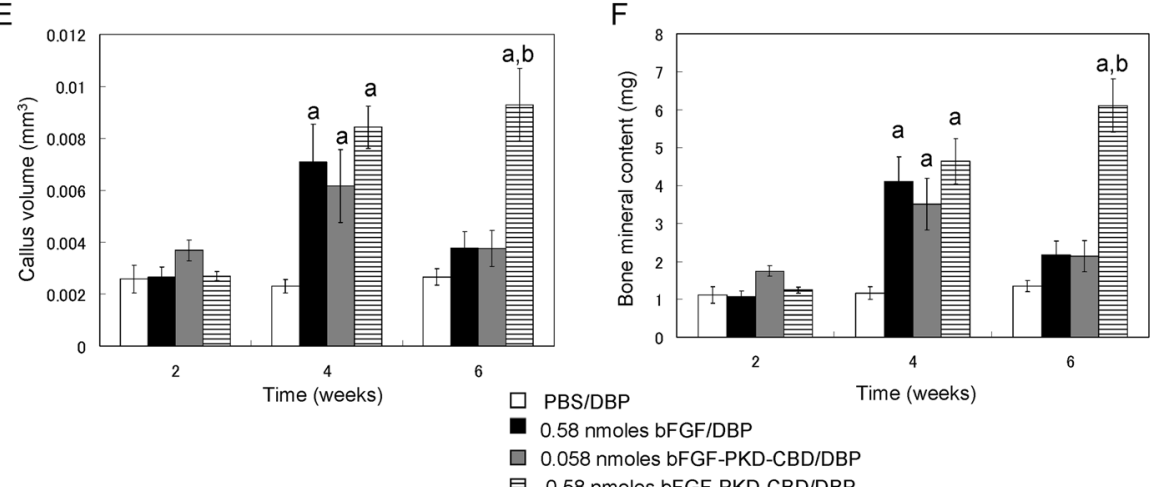

$\mathrm{F}$

$0.58 \mathrm{nmoles} b F G F-P K D-C B D / D B P$

Figure 2 3D micro- $C T$ analysis of femurs after grafting of demineralized allogenic bone powder loaded with bFGF-PKD-CBD. 3D micro-CT images of fractured mouse femurs treated with (A) PBS/DBP, (B) 0.58 nmol bFGF/DBP, (C) $0.058 \mathrm{nmol}$ bFGF-PKD-CBD/DBP, and (D) $0.58 \mathrm{nmol}$ bFGF-PKD-CBD/ DBP at 6 weeks. Green: newly formed bone; gray: existing bone. The scale bars indicate $3 \mathrm{~mm}$. (E) Callus area and (F) bone mineral content at fracture sites for the indicated treatment groups. Data are presented as the mean \pm standard error (S.E.; error bars; $n=6$ ). a: $P<0.05$ compared with the control group (PBS/DBP). b: $P<0.05$ compared with the $0.58 \mathrm{nmol}$ bFGF group.

PBS/DBP (Figure 2E, F). After 6 weeks, the callus volume and bone mineral content in fracture sites grafted with $0.58 \mathrm{nmol}$ bFGF-PKD-CBD/DBP were significantly higher than those found in the other three groups of treated femurs. In addition, no differences in callus formation or bone mineral content in fracture sites treated with PBS/ DBP, bFGF/DBP, or $0.058 \mathrm{nmol}$ bFGF-PKD-CBD/DBP femur were observed at 6 weeks (Figure 2E, F).

\section{Discussion}

In mice, femur fracture sites grafted with $0.58 \mathrm{nmol}$ bFGF-PKD-CBD/DBP had significantly higher levels of callus volume and bone mineral content at 4 weeks compared to fractures treated with DBP alone. In addition, treatment with a lower concentration of bFGF-PKD-CBD/ DBP than that used for bFGF/DBP led to higher comparative callus volume and bone mineral content compared to fractures treated with DBP alone at 4 weeks. Notably, the callus volume and bone mineral content in fracture sites grafted with $0.58 \mathrm{nmol}$ bFGF-PKD-CBD/DBP at 6 weeks were higher than those of the other three treatment groups. Taken together, these results suggest that bFGF$\mathrm{PKD}-\mathrm{CBD} / \mathrm{DBP}$ may be useful for promoting fracture healing in the clinical setting.

Demineralized bone material is a useful bone-filling material due to its excellent BMP-2 retention/liberation properties, which promotes cell-mediated mineralized matrix deposition [22,23]. However, implanting DBM alone for the reconstruction of bone defects often fails to achieve repair [7]. To overcome this limitation, Aspenberg et al. [24] mixed allograft bone with bFGF and showed that this combination increased the calcium content of bone after implantation into defect sites in rats. Lu and Rabie [25] also demonstrated that the combination of allograft bone and bFGF accelerates bone formation in rabbit. We previously established a method for accelerating periosteal bone formation using autologous DBP decorated with collagen-binding bFGF protein fused with a PKD domain and CBD derived from $C$. histolyticum class II collagenase $(\mathrm{ColH})$. In the present study, we demonstrated that the bFGF-PKD-CBD/DBP composite accelerates callus formation in a bone fracture model in mice and clearly showed that the composite also significantly increases the bone mineral density at fracture sites compared to bFGF/DBP. Notably, the callus volume and bone mineral content of fracture sites in the 0.58 nmol bFGF/DBP and 0.058 nmol bFGF-PKD-CBD/DBP treatment groups had decreased at 6 weeks compared to 4 weeks. This decrease was attributable to bone remodeling, which occurred when bone formation reached a plateau during the fracture healing process at approximately 4 weeks in these two treatment groups. In contrast, the callus volume and bone mineral content of femurs in the $0.58 \mathrm{nmol}$ bFGF-PKD-CBD/DBP group increased throughout the 6-week recovery period, suggesting that the bFGF-PKD-CBD/DBP composite continuously 
stimulated bone formation through its increased retention at fracture sites due to the presence of the PKD and CBD.

In addition, bFGF-PKD-CBD/DBP increased callus volume and bone mineral content to similar levels in fractures treated with a tenfold higher amount of bFGF at 4 weeks. Taken together, these findings suggest that bFGF-PKD-CBD/DBP composite has greater fracture healing effects than bFGF alone, even at markedly lower concentrations. This property may therefore reduce the adverse effects of bFGF, such as thrombocytopenia, renal toxicity, and malignant cell activation $[15,16]$, when used in the clinical setting.

During fracture healing, periosteal mesenchymal cells proliferate in the early phase and then differentiate into osteoblasts, which undergo expansion in the late phases and are involved in the synthesis of new bone. In the osteoinductive process, bFGF and BMP-2 play important roles in periosteal proliferation and osteogenic differentiation, respectively [26]. The osteogenic-promoting effects of synthetic bFGF and BMP-2 have also been reported [27-29]. For example, Akita et al. [27] showed that coadministration of BMP-2 and bFGF accelerates cranial bone defect healing. The combined delivery of BMP-2 and bFGF in the form of nanostructured colloidal gelatin gel also promotes bone regeneration in a rat femoral condyle defect model [28]. These findings suggest that the allogenic DBM/bFGF-PKD-CBD composite may stimulate both periosteal mesenchymal cell proliferation in the early phase of fracture healing due to the bFGF component and osteogenic differentiation in late phases due to the BMP-2 properties of DBM, leading to efficient fracture repair. Thus, allogenic DBM/ bFGF-PKD-CBD is a promising composite material for accelerating bone formation during fracture healing in the clinical setting.

\section{Conclusions}

We revealed that the anchoring of bFGF to demineralize allogenic DBP using the PKD and CBD derived from $C$. histolyticum collagenase effectively promotes fracture healing when this composite is applied to fracture sites. Specifically, the combination of DBP and the bFGFPKD-CBD fusion protein increased callus volume and bone mineral content when grafted onto fractured mice femurs. bFGF-PKD-CBD/DBP composite is therefore a promising agent for promoting fracture healing in the clinical setting.

\section{Competing interests}

The authors declare that they have no competing interests.

\section{Authors' contributions}

$\mathrm{KU}, \mathrm{OM}$, and MT designed the study and performed the analysis of the data. WS and KU wrote the manuscript. GI, HS, JA, and HF participate in the data collection. All authors read and approved the final manuscript.

\section{Acknowledgements}

This investigation was supported in part by Grants-in-Aid from the Ministry of Education, Sports, Culture, Science, and Technology of Japan to OM, MT, WS, and KU, by a Grant-in-Aid from the Japanese Ministry of Health, by a Medical Research Grant from The General Insurance Association of Japan, by a Kitasato University Research Grant for Young Researchers, and by research grants from the Parents' Association of Kitasato University School of Medicine.

\section{Author details}

${ }^{1}$ Department of Orthopaedic Surgery, Kitasato University School of Medicine, 1-15-1 Minami-ku Kitasato, Sagamihara, Kanagawa, Japan. ²Department of Bacteriology, Okayama University Graduate School of Medicine, Dentistry and Pharmaceutical Sciences, 2-5-1 Kita-ku Shikata-cho, Okayama, Japan.

Received: 19 February 2015 Accepted: 20 March 2015

Published online: 09 May 2015

\section{References}

1. Finkemeier CG. Bone-grafting and bone-graft substitutes. J Bone Joint Surg Am. 2002;84-A:454-64.

2. Heckman JD, Sarasohn-Kahn J. The economics of treating tibia fractures. The cost of delayed unions. Bull Hosp Jt Dis. 1997:56:63-72.

3. Rose FR, Oreffo RO. Bone tissue engineering: hope vs hype. Biochem Biophys Res Commun. 2002:292:1-7.

4. Ludwig SC, Boden SD. Osteoinductive bone graft substitutes for spinal fusion: a basic science summary. Orthop Clin North Am. 1999:30:635-45.

5. Peel SA, Hu ZM, Clokie CM. In search of the ideal bone morphogenetic protein delivery system: in vitro studies on demineralized bone matrix, purified, and recombinant bone morphogenetic protein. J Craniofac Surg. 2003:14:284-91.

6. Tiedeman JJ, Garvin KL, Kile TA, Connolly JF. The role of a composite, demineralized bone matrix and bone marrow in the treatment of osseous defects. Orthopedics. 1995:18:1153-8.

7. Chen B, Lin H, Wang J, Zhao Y, Wang B, Zhao W, et al. Homogeneous osteogenesis and bone regeneration by demineralized bone matrix loading with collagen-targeting bone morphogenetic protein-2. Biomaterials. 2007:28:1027-35.

8. Kale AA, Di Cesare PE. Osteoinductive agents. Basic science and clinical applications. Am J Orthop (Belle Mead NJ). 1995;24:752-61.

9. Friedlaender GE. OP-1 clinical studies. J Bone Joint Surg Am. 2001;83-A Suppl 1:S160-1.

10. Friedlaender GE, Perry CR, Cole JD, Cook SD, Cierny G, Muschler GF, et al. Osteogenic protein-1 (bone morphogenetic protein-7) in the treatment of tibial nonunions. J Bone Joint Surg Am. 2001:83-A Suppl 1:S151-8.

11. Govender S, Csimma C, Genant HK, Valentin-Opran A, Amit Y, Arbel R, et al. Recombinant human bone morphogenetic protein-2 for treatment of open tibial fractures: a prospective, controlled, randomized study of four hundred and fifty patients. J Bone Joint Surg Am. 2002;84-A:2123-34.

12. Kawaguchi $H$, Oka H, Jingushi $S$, Izumi T, Fukunaga M, Sato K, et al. A local application of recombinant human fibroblast growth factor 2 for tibial shaft fractures: a randomized, placebo-controlled trial. J Bone Miner Res. 2010;25:2735-43.

13. Kigami R, Sato S, Tsuchiya N, Yoshimakai T, Arai Y, Ito K. FGF-2 angiogenesis in bone regeneration within critical-sized bone defects in rat calvaria. Implant Dent. 2013;22:422-7.

14. Lai RF, Zhou ZY, Chen T. Accelerating bone generation and bone mineralization in the interparietal sutures of rats using an rhBMP-2/ACS composite after rapid expansion. Exp Anim. 2013;62:189-96.

15. Epstein SE, Fuchs S, Zhou YF, Baffour R, Kornowski R. Therapeutic interventions for enhancing collateral development by administration of growth factors: basic principles, early results and potential hazards. Cardiovasc Res. 2001:49:532-42.

16. Unger EF, Goncalves L, Epstein SE, Chew EY, Trapnell CB, Cannon III RO, et al. Effects of a single intracoronary injection of basic fibroblast growth factor in stable angina pectoris. Am J Cardiol. 2000;85:1414-9.

17. Nishi N, Matsushita O, Yuube K, Miyanaka H, Okabe A, Wada F. Collagen-binding growth factors: production and characterization of functional fusion proteins having a collagen-binding domain. Proc Natl Acad Sci U S A. 1998;95:7018-23.

18. Uchida K, Matsushita O, Naruse K, Mima T, Nishi N, Hattori S, et al. Acceleration of periosteal bone formation by human basic fibroblast growth 
factor containing a collagen-binding domain from Clostridium histolyticum collagenase. J Biomed Mater Res A. 2014;102:1737-43.

19. Saito W, Uchida K, Ueno M, Matsushita O, Inoue G, Nishi N, et al. Acceleration of bone formation during fracture healing by injectable collagen powder and human basic fibroblast growth factor containing a collagen-binding domain from Clostridium histolyticum collagenase. J Biomed Mater Res A. 2014;102:3049-55.

20. Katano M, Naruse K, Uchida K, Mikuni-Takagaki Y, Takaso M, Itoman M, et al. Low intensity pulsed ultrasound accelerates delayed healing process by reducing the time required for the completion of endochondral ossification in the aged mouse femur fracture model. Exp Anim. 2011;60:385-95.

21. Uchida K, Urabe K, Naruse K, Kozai Y, Onuma K, Mikuni-Takagaki Y, et al. Differential age-related bone architecture changes between female and male STR/Ort mice. Exp Anim. 2012;61:59-66.

22. Tuli SM, Singh AD. The osteoninductive property of decalcified bone matrix. An experimental study. J Bone Joint Surg (Br). 1978;60:116-23.

23. Wittbjer J, Palmer B, Rohlin M, Thorngren KG. Osteogenetic activity in composite grafts of demineralized compact bone and marrow. Clin Orthop Relat Res. 1983. 229-238.

24. Aspenberg $P$, Thorngren KG, Lohmander LS. Dose-dependent stimulation of bone induction by basic fibroblast growth factor in rats. Acta Orthop Scand. 1991;62:481-4

25. Lu M, Rabie AB. The effect of demineralized intramembranous bone matrix and basic fibroblast growth factor on the healing of allogeneic intramembranous bone grafts in the rabbit. Arch Oral Biol. 2002;47:831-41.

26. Wang L, Huang Y, Pan K, Jiang X, Liu C. Osteogenic responses to different concentrations/ratios of BMP-2 and bFGF in bone formation. Ann Biomed Eng. 2010;38:77-87.

27. Akita S, Fukui M, Nakagawa H, Fujii T, Akino K. Cranial bone defect healing is accelerated by mesenchymal stem cells induced by coadministration of bone morphogenetic protein-2 and basic fibroblast growth factor. Wound Repair Regen. 2004;12:252-9.

28. Su J, Xu H, Sun J, Gong X, Zhao H. Dual delivery of BMP-2 and bFGF from a new nano-composite scaffold, loaded with vascular stents for large-size mandibular defect regeneration. Int J Mol Sci. 2013;14:12714-28.

29. Wang H, Zou Q, Boerman OC, Nijhuis AW, Jansen JA, Li Y, et al. Combined delivery of BMP-2 and bFGF from nanostructured colloidal gelatin gels and its effect on bone regeneration in vivo. J Control Release. 2013;166:172-81.

\section{Submit your next manuscript to BioMed Central and take full advantage of:}

- Convenient online submission

- Thorough peer review

- No space constraints or color figure charges

- Immediate publication on acceptance

- Inclusion in PubMed, CAS, Scopus and Google Scholar

- Research which is freely available for redistribution 